\title{
Modelling of Stimulation Environment Using Monophasic Rectangle Pulse for Various Stimulation Parameters
}

\author{
M.E. CELIK* AND I. KARAGOZ \\ Gazi University, Department of Electrical-Electronics Engineering, Ankara, Turkey
}

\begin{abstract}
Recently, both the progress in some technological fields and multidisciplinary studies conducted with collaboration from different branches of science have impressive effect on clinically tested systems. Studies on development of visual prosthesis, which is based on passing damaged parts of the visual pathway and electrically stimulating nerve cells remaining intact, date back to elicit visual sense in blind patients. Investigation of some research topics, in silico, which should be taken into consideration in the design phase before expensive animal experiments provides great advantages in terms of both financial and time issues. In this study, factors such as heat, electric field distribution for current thresholds, current density which should be discussed in the design phase are simulated using monophasic rectangle pulses depending on various stimulation parameters with developed computational retina model. Change of heat, electric field, current density in points selected from center and periphery of retina tissue are investigated for various stimulation parameters. As a result, it is concluded that distribution of heat and electric field intensity over the periphery retina are much less than center region. Moreover, when larger pulse width is used, change of heat and electric field intensity seems much more in regions from center retina near stimulation electrode. Current density is higher in the sharp ends of the electrode than flat regions. Besides, when the size of stimulation electrode increases, electric field distribution becomes more uniform.
\end{abstract}

DOI: 10.12693/APhysPolA.128.B-297

PACS: $87.85 . \mathrm{Tu}, 87.85 .-\mathrm{d}$

\section{Introduction}

Vision in healthy eye initiates with the light coming to the eye and results with the formation of the image in the brain with parallel feedback systems. Visual nerve network provides that light coming from outside is converted signal forms suitable for nerve cells in very limited time intervals thanks to complex multi-connected branches [1]. From the first layer of the retina tissue, retinal pigment epithelium (RPE), to last layer, retinal ganglion cell (RGC), vision evolves from localized light percepts to images with specific color, depth. Visual sense may be lost due to various diseases which damages to the visual system. Depending on the disease, converting electrical signal to the suitable form may not be successful or the signals cannot be delivered to the brain. Retinitis pigmentosa (RP) and Age related macular degeneration (AMD) are the most encountered degenerative eye diseases causing unavoidable blindness. There are 40 million blind people and $82 \%$ of them are over 50 years old according to the 2010 data of World Health Organization [2]. It is reported that the most common eye disease causing blindness over 80 years old is AMD and its incidence rate is $25 \%$ in developed countries [3]. The aim of the visual prosthesis system is to provide visual perception by stimulating remaining intact nerve tissues or bypassing damaged parts $[4,5]$.

In this study, electrical stimulation of the retina model are simulated using monophasic rectangle pulses, which

*corresponding author; e-mail: mahmutemincelik@gazi.edu.tr is one of the most commonly used stimulation methods in experiments with animal and human subjects. After modelling retina and stimulation pattern, some parameters are examined, such as electric field, current density, temperature, in different points of the retina. These parameters are of prime importance for designing a visual prosthesis and could provide foresight in the design phase of a system.

\section{Methodology for developed model}

Dimensions of 2 dimensional retina model are determined as width of $2 \mathrm{~mm}$ and height of $200 \mu \mathrm{m}$ in harmony with real one, as shown in Fig. 1. It is assumed that retina is homogeneous and isotropic. Stimulation and ground electrode are placed to retinal ganglion cell layer and retinal pigment epithelium layer respectively, which corresponds to epiretinal stimulation concept. Voltage difference is applied between epiretinally placed electrodes. Three different points which are determined over the retina for representing three main regions of the retina are used to compare regional differences. While first probe is close to the stimulation electrode and represents retinal ganglion cell layer, second probe represents inner cell layers. Additionally, third probe is placed near to retinal pigment epithelium layer and ground electrode.

The analysis include time-dependent electric field distribution in two dimensional space. While the Maxwell equations and their subsets form a basis for modeling formulations, differential forms are solved by finite element analysis method and predefined edge element discretization algorithms.

Electric field distribution is firstly examined for three different points. Then, it is examined depending on different widths of monophasic pulse, which are $0.2,0.3$ 


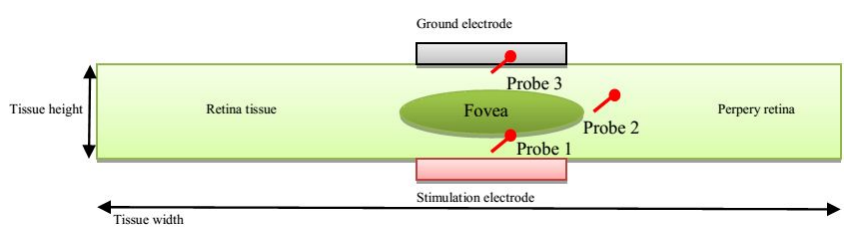

Fig. 1. Retina model involving different measurement regions.

and $0.4 \mathrm{~ms}$. Next, current density is also observed from beginning to $0.7 \mathrm{~ms}$ of the pulse. On the other hand, temperature change, which is induced by power dissipated from stimulation electrode, is analysed for three points.

Heat transfer is based on the Pennes bioheat equation by $(1)$

$$
\nabla(-k \nabla T)=\rho_{b} C_{b} \omega_{b}\left(T_{b}-T\right)+Q_{m e t}+Q_{e x t},
$$

where $k, \rho_{b}, C_{b}, \omega_{b}, Q_{m e t}, Q_{e x t}, k \rho_{b}, C_{b} \omega, Q_{m e t}, Q_{e x t}$ represent thermal conductivity $(\mathrm{W} / \mathrm{mK})$, density $\left(\mathrm{kg} / \mathrm{m}^{3}\right)$, specific heat capacity which is the amount of energy to produce change of a unit temperature $(\mathrm{J} / \mathrm{kg} \mathrm{K})$, perfusion rate which shows volume of blood per second flowing through a unit volume of tissue $(1 / \mathrm{s})$, heat source of metabolism and external heat source $\left(\mathrm{W} / \mathrm{m}^{3}\right)$ respectively.

Voltage distribution for the model is governed by Poisson equation given by

$$
-\nabla\left(\sigma_{b} \nabla V_{e}\right)=I,
$$

where $\sigma_{b}$ represents conductivity of medium, taken as 1 , and $I\left[\mathrm{~A} / \mathrm{m}^{3}\right]$ is current density which is applied. Equations coupling intracellular and extracellular potentials for further studies are formalized as Eq. (3) and (4)

$$
\begin{aligned}
& \sigma_{e} \nabla^{2} V_{e}=I_{m}, \\
& \sigma_{i, z} \frac{\partial^{2} V_{i}}{\partial z^{2}}=I_{m},
\end{aligned}
$$

where $V_{i}, V_{e}, \sigma_{i, z}, \sigma_{e}$ and $I_{m}$ are intracellular, extracellular voltage and conductivity and membrane current. The model includes current conservation rule and electric insulation with equations given by Eqs. (5)-(8) respectively for time dependent model.

$$
\begin{aligned}
& \nabla J=Q_{j}, \\
& J=\left(\sigma+\varepsilon_{0} \varepsilon_{r} \frac{\partial}{\partial t}\right) E+J_{e}, \\
& E=-\nabla V, \\
& \boldsymbol{n} \cdot \boldsymbol{J}=0,
\end{aligned}
$$

where $J, Q_{j}, \varepsilon_{r}, E$ represent respectively current density $\left[\mathrm{A} / \mathrm{m}^{2}\right]$, charge, relative permittivity and electric field $[\mathrm{V} / \mathrm{m}]$.

\subsection{Investigation of electric field distribution and current density and temperature change}

Electric field forms due to voltage difference applied between stimulation and ground electrode. Fig. 2A shows electric field distribution for three different probes to compare intensities among different regions of the retina.
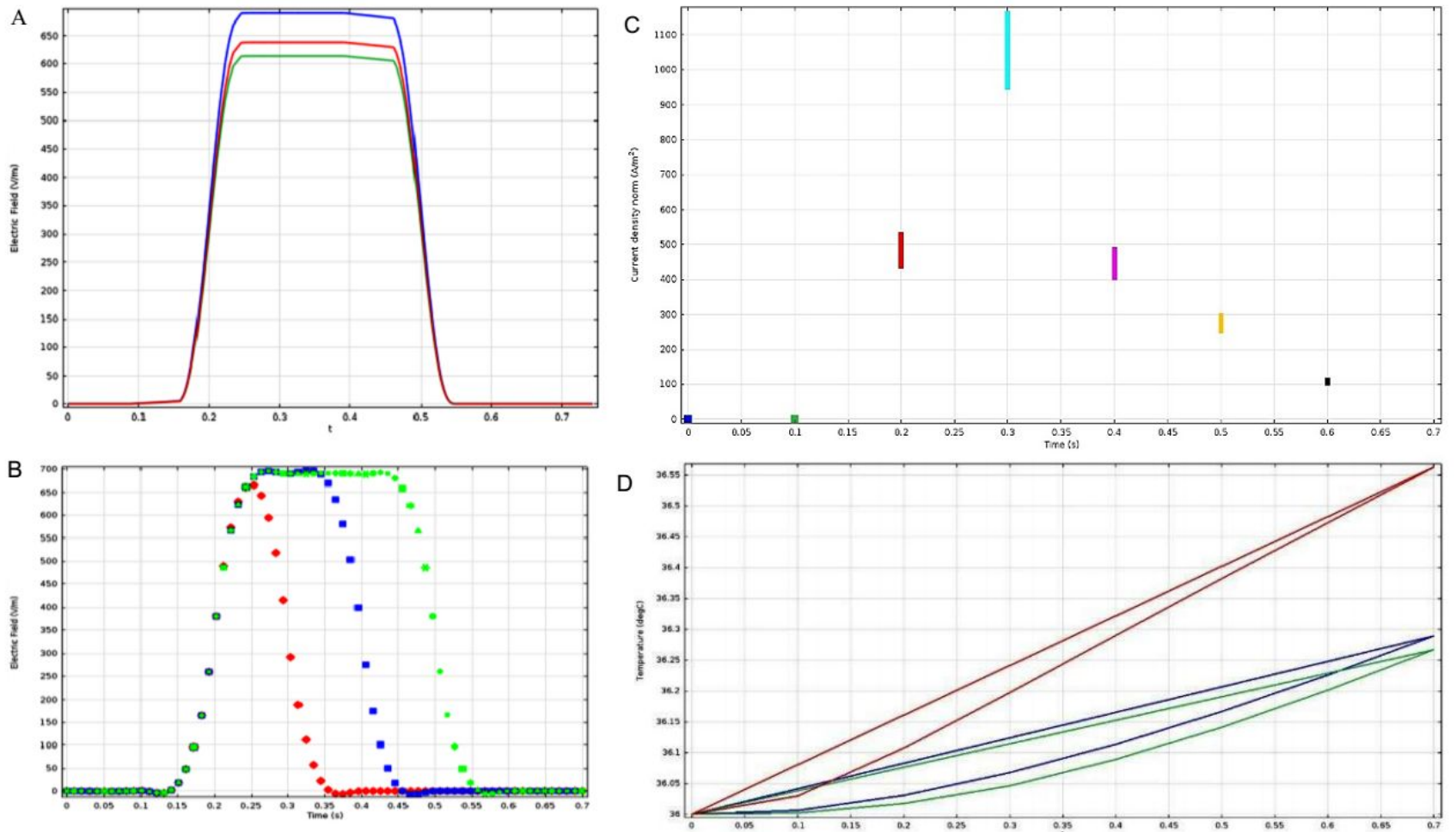

Fig. 2. Electric field distribution over the retina for three probes (A), electric field distribution depending on different pulse widths, $0.2,0.3,0.4 \mathrm{~ms}$. (B), distribution of current density norm $(\mathrm{C})$, temperature change (D). 
It is observed from blue, red and green lines, which indicate first, third and second probes result that maximum electric field intensity is near the stimulation electrode because it is generated by the source, stimulation electrode. As it is moved from the source to periphery regions intensity quietly decreases. Larger pulse widths provide extended period of time of maximum pulse, as shown in Fig. 2B. Figure 2C demonstrate that as the potential difference of the pulse increases, the current density also increases. Current density is much more in sharp edges of the electrodes. Because of power dissipated due to stimulation electrode, heat generates and temperature changes. In Fig. 2D, red, blue and green indicates probe 1 , probe 3 and probe 2 respectively, so maximum temperature change of them are $0.57^{\circ} \mathrm{C}, 0.29^{\circ} \mathrm{C}, 0.26^{\circ} \mathrm{C}$. Temperature is much high near the source and the region between electrodes. It is seen that periphery regions are less affected.

Electrical stimulation is of major importance for development of visual prosthesis. One of the most significant parameters to examine electrical stimulation effect is stimulation threshold. It is defined as the lowest stimulation amplitude that predefined success rate is satisfied for all trials. The performance of electrical stimulation mainly depends on electrode and stimulation parameters.

Electrode parameters include electrode geometry, electrode size, distance to retina tissue and ground electrode position. Electrode geometry affects remarkably electric field distribution over retina tissue. Ideal stimulation is required ganglion cell size stimulation, which is cell size electrode. So, electrode crosstalk is diminished with localized stimulation. Localized stimulation is also required that ground electrode should be placed same side with stimulation electrodes. Efficient electrical stimulation is directly proportional to distance between electrode and retina tissue. As it is moved away from tissue, stimulation threshold increases exponentially.

On the other hand, stimulation parameters involve stimulation amplitude, pulse width or duration and pulse polarity. Stimulation which has certain limitations is required for safe and long term routine. These limitations provide to avoid tissue damage and electrode corrosion. Stimulating retina ganglion cells and nervous activity could only be possible appropriate pulse width. Longer pulse widths enables lower driving voltage. Cathodicfirst stimulations is efficient in terms of providing direct cell depolarization.

\section{Conclusion}

There are some physical, electrical and biological constraints that should be taken into consideration for an efficient visual prosthesis which will be used long term. It is quite risky in terms of usage that the implants which are placed to the retina and stay there through long-term induce high temperature change.

This study showed that monophasic rectangle pulses with various stimulation parameters have an important role to induce spike activity by enabling electric field over retina ganglion cells. Safe and long term stimulation could be possible under certain limitations. Similarly, heat change could be allowable range when retina is stimulated with monophasic rectangle pulses. Simulation models are powerful tool and used to understand how a visual prosthesis is designed properly, and which parameter affects the performance of the system crucially before it is fabricated. Because clinical trials on humans extend to the experimental procedure, development of human-independent modelling, simulation and experimental work will have great importance in the future.

\section{References}

[1] J.D. Weiland, M.S. Humayun, IEEE Trans. Biomed. Engin. 61, 1412 (2014).

[2] D. Plankar, A. Vankov, P. Huie, S. Baccus, J. Neural Engin. 2, 105 (2005).

[3] M.S. Humayun, J.D. Weiland, G.Y. Fujii, R. Greenberg, R. Williamson, J. Little, B. Mech, V. Cimmarusti, G. Van Boemel, G. Dagnelie, E. de Juan, Vision Res. 43, 2573 (2003).

[4] E. Zrenner, A. Stett, S. Weiss, R.B. Aramant, E. Guenther, K. Kohler, K.D. Miliczek, M.J. Seiler, H. Haemmerle, Vision Res. 39, 2555 (1999).

[5] J.D. Weiland, A.K. Cho, M.S. Humayun, Ophthalmology 118, 2227 (2011). 